\title{
SHALLOW-DEEP INSTABILITY OF DONOR STATES IN MANY-VALLEY SEMICONDUCTORS
}

\author{
M. GRINBERG \\ Institute of Physics, N. Copernicus University \\ ul. Grudziądzka 5/7, 87-100 Toruń, Poland
}

The shallow-deep instability of double and single donors in multi-valley semiconductors using the model Hamiltonian is studied. The obtained results describe properly the properties of double donors (in $D^{0}$ and $D^{+}$state) in Ge and single deep donors in $\mathrm{Al}_{x} \mathrm{Ga}_{1-x}$ As for $0.3<x<0.5$.

PACS numbers: 71.55.-i, 71.38.+i

The deep donor centres are well known in $\mathrm{Si}$ and $\mathrm{Ge}$ [1] as resulting from doping with $\mathrm{O}, \mathrm{S}, \mathrm{Se}$ and Te-the impurities which form the double donors in these materials. Also the single donors $\mathrm{Si}$ or $\mathrm{S}$ in $\mathrm{Al}_{x} \mathrm{Ga}_{1-x} \mathrm{As}$ for $x$ between 0.3 and 0.5 may introduce the localized deep levels in the middle of the gap (DX centres). It seems to be proved that the lattice relaxation determines many properties of the DX, however deep donor levels in AlGaAs without the relaxation of the lattice were also reported [2]. Although hundreds of experimental and theoretical papers concerning the DX centres were published we are still far from the complete understanding of their microscopic structure [3-7]. Therefore it is particularly interesting which properties of the deep donors may result from the electronic interactions. This is a main reason why the calculations presented in this paper were intentionally restricted to consider only the electronic part of the Hamiltonian. The problem of the electron-phonon interaction was discussed elsewhere $[9,10]$.

The model Hamiltonian used in this paper is similar to that proposed by Resca [11]. However, it is more realistic since it describes the semiconductor with four equivalent minima of $L$ conduction band (actual structure which determines the properties of donor states in $\mathrm{Ge}$ and $\mathrm{Al}_{x} \mathrm{Ga}_{1-x} \mathrm{As}$ for $0.3<x<0.5$ ). The second difference comes from the approximation in which the kinetic energy of electron outside the Brillouin zone is given by the dispersion relation of the free electron. Assuming that the dispersion around each minimum is defined by the electron effective mass $m$ one obtains the following relation on the kinetic energy term:

$$
T(k)= \begin{cases}\sum_{j} \hbar^{2} \frac{\left(k-K_{j}\right)^{2}}{2 m}, & \left|k-K_{j}\right|<K_{\mathrm{B}}, \\ \hbar^{2} K_{\mathrm{B}}^{2} / 2 m, & \left|K_{j}+K_{\mathrm{B}}\right|>\left|k-K_{j}\right|>K_{\mathrm{B}} \\ \hbar^{2} k^{2} / 2 m_{0}-E_{0}, & k>\left|K_{j}+K_{\mathrm{B}}\right|\end{cases}
$$


Here $\boldsymbol{K}_{\boldsymbol{j}}$ vectors define the positions of the minima in the Brillouin zone, $\boldsymbol{K}_{\mathrm{B}}$ was calculated from the width of the conduction band and $m_{0}$ is the free electron mass. The potential energy term is given by the sum of long-range point charge potential $V_{\mathrm{pc}}$ [12] and the local potential described by the depth $V_{1}$ and the radius $R_{1}$. It was discussed in [10] that this type of Hamiltonian describe the localized as well as the delocalized states. Parameters: $K_{i}, K_{\mathrm{B}}, m, E_{0}$ and dielectric function describe the semiconductor whereas $V_{l}$ and $R_{l}$ depend on the donor.

The calculations were performed in the framework of variational method with one-electron trial functions given by the symmetrized combinations of Gaussians with one variation parameter $a$ :

$$
F_{n}(r)=\sum_{j} \alpha_{n}^{j} \exp \left(-\mathrm{i} K_{j} \cdot r\right) \exp \left(-r^{2} / 2 a^{2}\right) /\left(\pi^{3 / 4} a^{3 / 4}\right)
$$

For double donors the Slater determinants constructed from all Pauli principle allowed pair functions (2) were used.

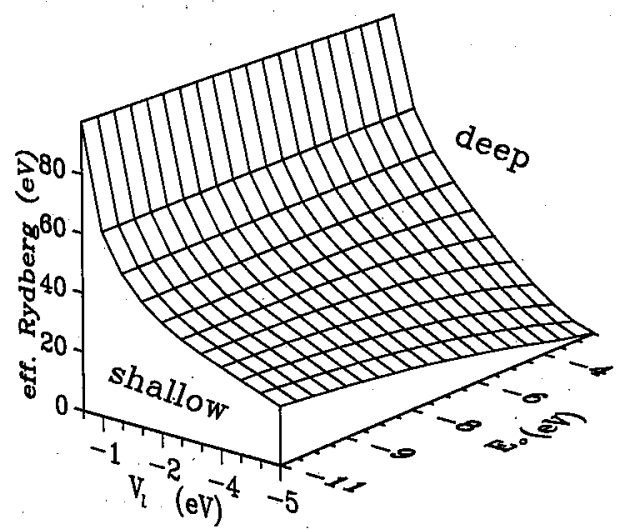

Fig. 1. The shallow-deep instability diagram for the single donors. Here instead of effective mass and dielectric constant $\varepsilon$ the effective Rydberg $R^{*}=\mathrm{Ry}{ }^{*} m / \varepsilon^{2}$ as an input parameter was used. $R_{\mathrm{I}}=4.1$ a.u. The values of $R^{*} 50 \mathrm{meV}$ for $L$ band in GaAs and $16.5 \mathrm{meV}$ for Ge yield the deep and shallow ground state of single donors, respectively.

For both single and double donors one may obtain the ground state energy as a functional of variational parameter $E(a)$. For the most interesting case, for the critical values of input parameters $E(a)$ has two minima, the first one, for large $a$ corresponding to the shallow donor state and the second one, for the small $a$ (approximately 5 a.u.) corresponding to the localized deep donor case. The type of the final state is determined by the deeper of the two minima. This criterion was used to analyze the effect of the shallow-deep instability. The results obtained for single donors are presented in Fig. 1 in the form of shallow-deep instability diagram. It should be mentioned here that when the $\Gamma$ minimum is considered the collapsing of thestate does not occur for any real values of $V_{1}$ and $R^{*}$. Therefore the shallow states pinned to the $\Gamma$ minimum exist independently 
on the deep state related to $L$ minima. For double donor, for the charge state $D^{+}$(one electron captured on donor), the shallow-deep diagram is very similar to presented in Fig. 1 (with $R^{*}$ scaled by factor about 0.25 ). For the neutral state $D^{0}$ the problem is more complicated. In order to consider this case let us see Fig. 2 where the energies of the ground states of $D^{0}$ and $D^{+}$versus the local potential $V_{I}$ (with other parameters taken as for $\mathrm{Ge}$ ) are presented. Depending on the local

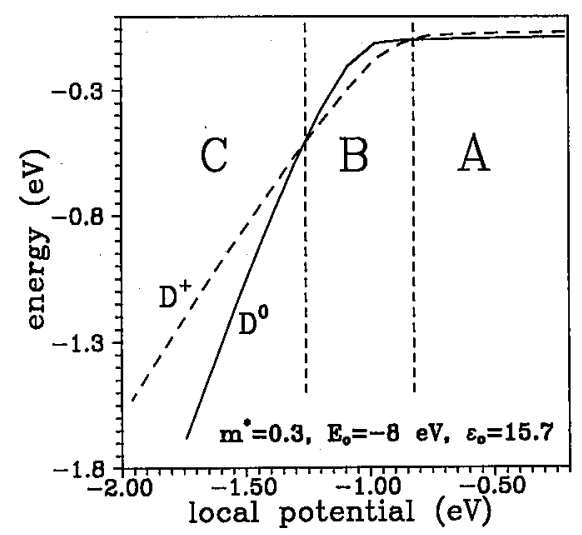

Fig. 2. The energy of the ground state of double donors versus the local potential depth $V_{1}, R_{1}=5$ a.u. $D^{0}-$ solid curve, $D^{+}-$dashed curve. The transition $D^{0} \rightarrow D^{+}$ corresponds to the ionization of one electron to the conduction band. The energy of the system with two electrons in conduction band is zero. The regions marked by $A, B$ and $\mathrm{C}$ respectively correspond to: A. For both $D^{+}$and $D^{0}$ all bound states are shallow (the electron wave functions spread over the tens of lattice constants). The spectrums of $D^{0}$ and $D^{+}$correspond to the helium-like spectrum $\mathrm{He}^{0}$ and $\mathrm{He}^{+}$, reduced by the effective Rydberg constant. Because the ground states are: the singlet $A_{l}$ for $D^{0}$ and doublet $A_{l}$ for $D^{+}$, the small chemical shifts should be observed. B. The ground state of $D^{+}$becomes localized, but the Coulomb interaction between electrons, still large in comparison with local potential, forbids the strong localization of the second electron. For $D^{0}$ the strong localization of only one electron is energetically favourable while the second electron is captured by the long-range Coulomb potential in the shallow state. $D^{0}$ spectrum is then hydrogen-like $\left(\mathrm{H}^{0}\right.$, shallow donor), whereas $D^{+}$has the deep ground state and $\mathrm{He}^{+}$-like excited shallow states. C. $D^{0}$ and $D^{+}$ground states are localized (the two deep donor states related to the double donor can be observed). The excited states are still shallow, corresponding to $H^{0}$-like and $\mathrm{He}^{+}$-like for $D^{0}$ and $D^{+}$, respectively.

potential the three types of defects, described in figure caption: $A, B$, and $C$, are possible. Actually almost all double donors in Ge [1] seem to represent $C$ type with two deep levels related to $D^{0}$ and $D^{+}$. It should be mentioned here that, for the localized states, the electron-phonon interaction is about two times larger for $D^{0}$ with two localized electrons than for $D^{+}$. This fact may result in the local lattice distortion which changes the order of $D^{+}$and $D^{0}$ ground states, so the defects of $B$ type must not be represented in the real crystal. 


\section{References}

[1] H.G. Grimmeiss, L. Montelius, K. Larsson, Phys. Rev. B 37, 6916 (1988) and the reference therein.

[2] See for example J.E. Dmochowski, R.A. Stradling, Int. Symposium on DX Centers and Other Metastable Defects in Semiconductors, 18-22 Feb. 1991, Mauterndorf (Austria), to be published in Semicond. Sci. Technol..

[3] T.N. Morgan, in [2].

[4] D.J. Chadi, K.J. Chang, Phys. Rev. Lett.61, 873 (1988), Phys. Rev. B 39, 10366 (1989).

[5] J. Dabrowski, M. Sheffler, R. Strehlow, in Physics of Semiconductors, Eds. E.M. Anastassakis, J.D. Joannopoulos, Vol. 1, 1990, p. 489.

[6] E. Yamagushi, K. Shiraishi, T. Ohno, in [5], p. 501.

[7] One may compare the results of calculations of Chadi et al. [4] or Dabrowski et al. [5] with Yamagushi et al. [6], where the different approximations of exchange potential yield the different predictions concerning the DX ground state. The quite different model of DX centers have been proposed recently by Morgan [3].

[8] G.A. Baraff, in [2].

[9] M. Grinberg, Phys. Rev. B 41, 8323 (1990).

[10] M. Grinberg in [2].

[11] L. Resca, D.J. Lohrman, Phys. Rev. B 36, 1741 (1987);

L. Resca, Can. J. Phys. 67, 896 (1989).

[12] R. Resta, Phys. Rev. B 16, 2717 (1977);

M. Grinberg, S. Legowski, H. Meczynska, Phys. Status Solidi B 130, 325 (1985). 